Document downloaded from:

http://hdl.handle.net/10251/159525

This paper must be cited as:

Arce Vila, P.; De Fez Lava, I.; Belda Ortega, R.; Guerri Cebollada, JC.; Ferrairó, S. (2019). Proxy-based near real-time TV content transmission in mobility over 4G with MPEG-DASH transcoding on the cloud. Multimedia Tools and Applications. 78(18):26399-26425. https://doi.org/10.1007/s11042-019-07840-6

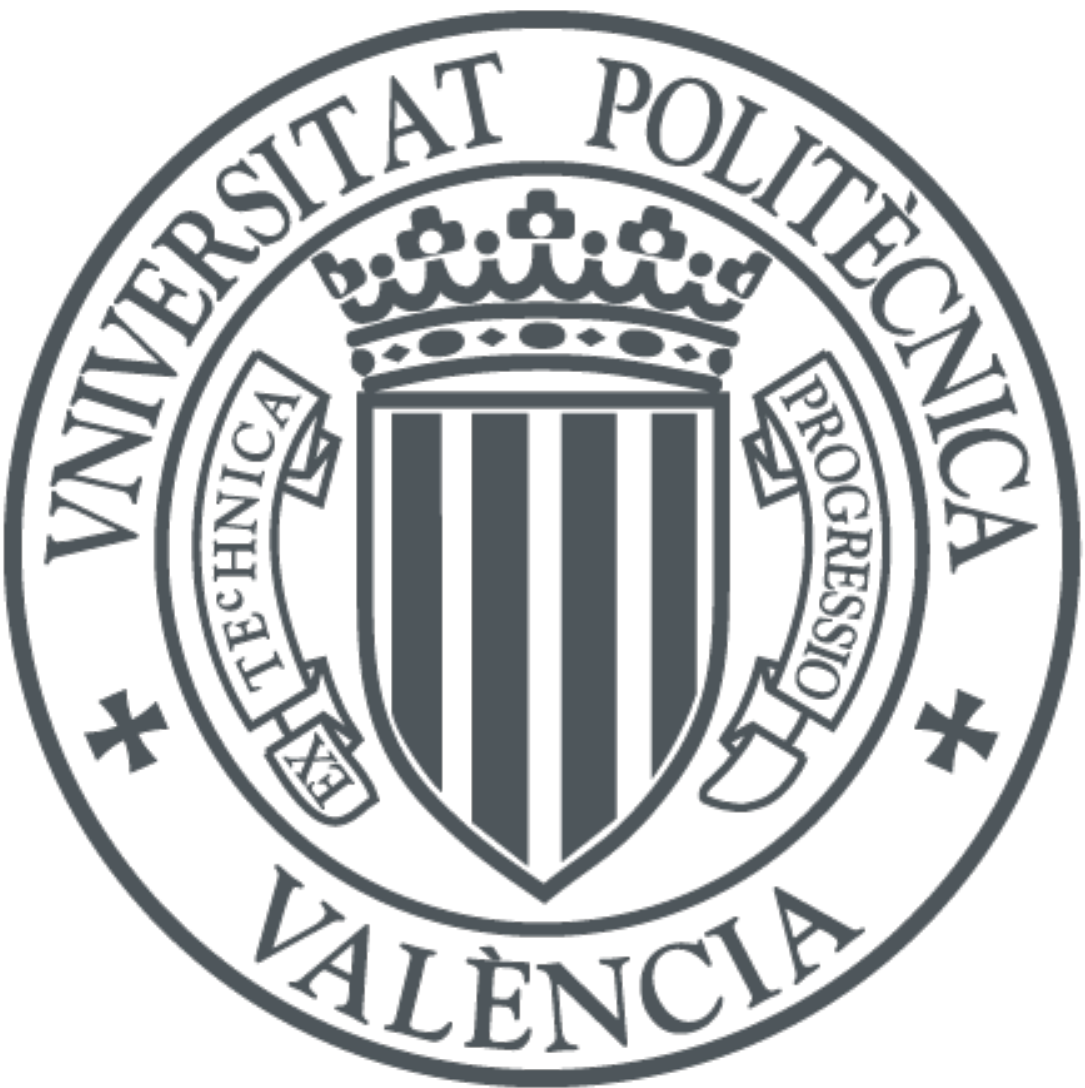

The final publication is available at

https://doi.org/10.1007/s11042-019-07840-6

Copyright Springer-Verlag

Additional Information 


\title{
Proxy-based near real-time TV content transmission in mobility over 4G with MPEG-DASH transcoding on the cloud
}

\author{
Pau Arce ${ }^{\mathrm{a}}$, Ismael de Fez ${ }^{\mathrm{a}}$, Román Belda ${ }^{\mathrm{a}}$, Juan Carlos Guerri ${ }^{\mathrm{a}}$, Salvador Ferrairóo ${ }^{\mathrm{b}}$ \\ a Institute of Telecommunications and Multimedia Applications (iTEAM), \\ Universitat Politècnica de València, Camino de Vera, 46022, Valencia, Spain \\ ${ }^{\mathrm{b}}$ Azimut Electronics, Carrer dels Furs 50, 46701, Gandia, Valencia, Spain \\ e-mail: paarvi@iteam.upv.es, isdefez@iteam.upv.es, robelor@iteam.upv.es, jcguerri@dcom.upv.es, \\ ferrairo@azimut.es \\ phone: $34-963879588$
}

\begin{abstract}
This paper presents and evaluates a system that provides TV and radio services in mobility using 4G communications. The system has mainly two blocks, one on the cloud and another on the mobile vehicle. On the cloud, a DVB (Digital Video Broadcasting) receiver obtains the TV/radio signal and prepares the contents to be sent through 4G. Specifically, contents are transcoded and packetized using the DASH (Dynamic Adaptive Streaming over HTTP) standard. Vehicles in mobility use their $4 \mathrm{G}$ connectivity to receive the flows transmitted by the cloud. The key element of the system is an on-board proxy that manages the received flows and offers them to the final users in the vehicle. The proxy contains a buffer that helps reduce the number of interruptions caused by hand over effects and lack of coverage. The paper presents a comparison between a live transmission using $4 \mathrm{G}$ connecting the clients directly with the cloud server and a near real-time transmission based on an on-board proxy. Results prove that the use of the proxy reduces the number of interruptions considerably and, thus, improves the Quality of Experience of users at the expense of slightly increasing the delay.
\end{abstract}

Keywords: Proxy, 4G, Video streaming, TV, Buffering, Dynamic Adaptive Streaming over HTTP (DASH), Quality of Experience, ITU-T P.1203

\section{Introduction}

It is well-known the fast growing of the Internet traffic in recent years, and forecasts indicate that this trend will continue in coming years. For example, according to the latest report carried out by Cisco in 2017 [1] global IP traffic will increase nearly threefold from 2016 to 2021.

Among the causes, we find, in first place, the access to the Internet of a growing number of people, mainly in the Middle East, Africa and Asia Pacific. In the most developed areas, the growing is due to the fact that users access an increasing number of applications and services throughout their mobile devices. The increase in Internet connection speeds, with the emergence of $4 \mathrm{G}$ technologies, has allowed users to consume bandwidth-demanding services (mainly video), what was not feasible until a few years ago.

In this way, users are in the habit of consuming video services using their mobile devices in any place and situation. Thus, it is quite common to see everyday people on the bus or on the subway watching a video in their mobile phones. Apart from the requirement of a great bandwidth inherent to video streaming, these are demanding 
environments since mobility represents an additional challenge. This problem becomes more important as the speed at which the user moves increases, for example, on long-distance road trips (either by bus or car), or on trains, boats and ferries.

In this environment, due to the importance of entertainment services nowadays, some transport companies have begun offering on-board entertaining platforms (films, TV shows, music, games...), which represent an added value in the transport service.

Only a few of these companies offer live TV and radio services, which involves the necessity to install a DVB receiving antenna as well as a server, which is in charge of transcoding the flows from the antenna and convert them to IP in order to be consumed by the users. The use of a DVB antenna results in a more expensive installation apart from the allocation of a physical space in a reduced environment. With the increasing speeds of $4 \mathrm{G}$ networks (and expecting the future arrival of $5 \mathrm{G}$ networks), it results affordable to offer TV services by means of $4 \mathrm{G}$ nowadays.

In any case, the main problem of live TV/radio in mobility is signal loss and the corresponding interruptions due to coverage cuts, e.g., when a bus goes through a tunnel. These interruptions undoubtedly degrade the Quality of Experience (QoE) of users, who are more and more demanding in their quality requirements for multimedia content consumption.

In order to solve this problem, this paper presents and evaluates a multimedia system that offers users in mobility $\mathrm{TV}$ and radio services in near real-time. To that end, the system uses advanced selective pre-storage strategies of content sent from transcoding servers located on the cloud. In this sense, one of the main contributions of the paper is a real implementation of the whole system and the configuration of the testbed, which is used to carry out the assessment of the proposal.

The rest of the paper is organized as follows. In Section 2, relevant related work is presented. Section 3 describes the proposed system architecture. Methodology is explained in Section 4. A thorough evaluation and results are shown in Section 5. In Section 6 some improvements to the key element of the proposal, the proxy, are presented. Finally, conclusions are discussed in Section 7.

\section{Related work}

One of the key elements in which this proposal is based on is the use of a proxy to manage the video flows received by $4 \mathrm{G}$. A proxy is, at the same time, a server and a client, i.e. a node that acts as an intermediary among clients and servers, retransmitting both requests and responses and potentially performing packet modifications, caching or filtering. Caching techniques and proxies have been widely used in communication networks (e.g. [2-3]), especially web proxies, a basic component of web caches, which help to reduce traffic considerably in the Internet.

Regarding the problem of explosive increasing in Internet traffic, different studies [4-7] state that an important part of multimedia traffic towards mobile devices is due to many downloads of only a few popular contents (e. g. musical videos) of large size. In this line, research is being focused on finding effective ways of reducing the transmission of duplicated contents by using smart caching strategies within mobile networks, thus allowing users to access popular contents by means of caches in nearby gateways of their operators. Examples can be seen in [8] and [9], where selective techniques of IP traffic based on traffic load or geolocation are used. Also, by reducing traffic load with the use of smart caching of popular content, the efficiency of $4 \mathrm{G}$ networks would improve. In 
fact, caching techniques both in $3 \mathrm{G}$ [4] and 4G [6] have managed to reduce traffic to mobile devices by 30 to 60 percent.

Additionally, it is worth highlighting some papers related to this proposal that analyze the use of proxies. For instance, [10] presents a mobile proxy architecture for improving the system performance over high-speed trains in Long Term Evolution Advanced (LTE-A) networks [11]. The proxy is used to save the wireless bandwidth consumption between the train and the base station, and also to reduce the starting time of the video. Another proposal based on the use of a proxy is shown in [12], which presents a proxy-based solution for adapting the scalable video streams at the edge of a wireless network. In that paper, the proxy helps to maximize the video quality of all receivers given a certain bandwidth. That solution is focused on scalable video coding (SVC). Talking about different video transmission techniques, [13] presents a system framework for network compression of interactive multi-view streaming video with path diversity. That system features a proxy-server, which is in charge of adapting the content data sent over the wireless links, with the aim of minimizing the video distortion at the client side. Finally, [14] proposes a DASH proxy-based bitrate algorithm for video communications over orthogonal frequency division multiple access (OFDMA) systems.

In the aforementioned articles, the evaluations are carried out by using on-demand video services in order to check the performance of the proposal. However, despite the amount of papers existing in the literature focused on proxies and caches, there is hardly related work publicly available in the literature about the use of proxies to offer live TV services, as this paper proposes. In this sense, although nowadays there are already some apps that provide live TV streaming, most of them are focused on providing video streaming services and do not pay special attention to network reliability, scalability or cost. In a scenario where, for instance, 100 users in the same location want to access the same TV channel simultaneously, it is possible that they have problems (in terms of connectivity, stalls, delays, etc.).

What has been widely studied is the idea of providing TV services in mobility. In this sense, one of the first standards aimed to provide TV for mobile devices was DVB-H (Digital Video Broadcasting - Handheld) [15], which was published in 2004 as a variation of DVB-T (Digital Video Broadcasting - Terrestrial) for mobile terminals. However, despite the characteristics of the standard, DVB-H did not success as expected, and nowadays it has barely been adopted. An evolution of DVB-H was DVB-SH (Digital Video Broadcasting - Satellite services to Handhelds) [16], published in 2007, which was based on a hybrid satellite/terrestrial downlink. Another family of standards to highlight is those proposed by 3GPP, especially MBMS (Multimedia Broadcast/Multicast Service) [17] and its evolution eMBMS (evolved MBMS) [18]. eMBMS was born as a technology to efficiently send the same content to a group of users in LTE networks. In fact, the jointly use of eMBMS and caching techniques can make transmission rather efficient. Although technologies like eMBMS can be used to boost the network's capability for providing high-quality multimedia service in high user-density area, the real fact is that this technology is not as wide spread as users would like to. In many locations and countries, mobile operators have not fully implemented it, or do not offer it in mobility.

In this sense, mobile services based on 4G LTE are continuously expanding in global markets today, providing subscribers with the same connection quality as it was previously only possible with broadband cable networks [19]. With more than 200 commercial LTE networks deployed worldwide in August 2013 [20], it is expected that LTE subscriptions will exceed 1300 million by the end of 2018 [21]. It is important to highlight the next generation of mobile telephony $5 \mathrm{G}$, which is expected to be implemented as of 2020 . The deployment of $5 \mathrm{G}$ networks will certainly improve the provision of multimedia content transmission services like the one presented in this paper, 
for instance, by providing video streaming at high resolutions (such as $4 \mathrm{~K}$ ). In this sense, satellites will play an important role in the extension of cellular networks by sea, air and remote areas not covered by small cellular networks [22]. In this way, with the use of satellites, the system hereby proposed could be used in other scenarios, such as on boats, which usually are located in areas without Internet coverage.

The capabilities of $4 \mathrm{G}$ regarding bandwidth have allowed to offer more demanding services, like streaming of high quality videos (e.g. 1080p or even $4 \mathrm{~K}$ ) or live TV. In this line, different studies have been published. For example, [23] proposes a framework to maximize radio resource usage when providing TV broadcasting over LTE networks, whereas [24] presents a resource allocation framework for adaptive video streaming over LTE based on the DASH standard. This work also aims at optimizing the use of the 4G network, as [23] and [24], but, in this case, the key element is a proxy installed in the on-board server. Moreover, our proposal is validated in a mobility scenario and not with simulations. Finally, it is worth highlighting different works that study the provision of TV services in mobility, such as [25] and [26]. [25] is mainly focused on the analysis of the physical channel (a hybrid solution of LTE and DTT -Digital Terrestrial Television- systems), and not in the QoE as our proposal does. On the other hand, [26] presents a new algorithm for transmitting multiple variable bitrate (VBR) video streams, which is focused on reducing energy consumption in mobile devices. Compared to our solution, that system is not intended for providing TV/radio services to a group of users located at the same place.

\section{System description}

\subsection{General scheme}

Fig. 1 shows a simplified scheme of the proposed system, which consists of two main blocks: the cloud and the shipped server in a vehicle. On the cloud, TV/radio contents are received by means of the DVB standard, and are transcoded and then transmitted through $4 \mathrm{G}$.

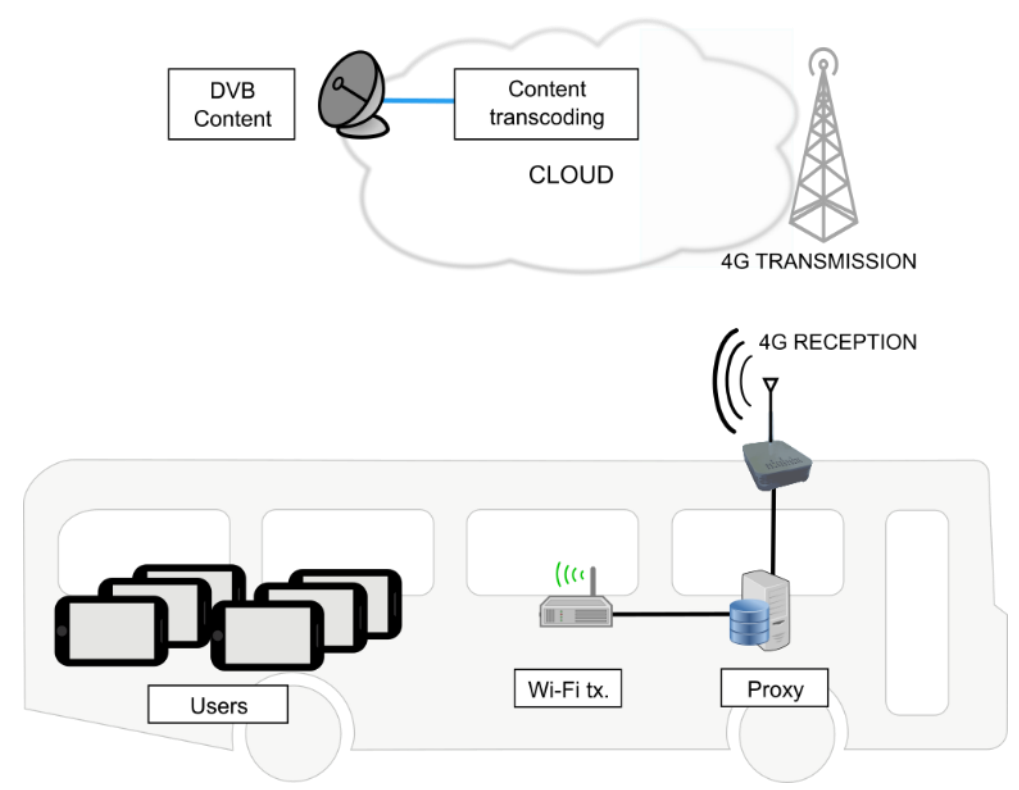

Fig. 1. Scheme of the proposed system

On the other hand, in the vehicle there is a $4 \mathrm{G}$ receiver, which receives the contents encoded on the cloud. Also, in the shipped server there is a proxy in charge of managing the reception of video packets from the cloud and, additionally, managing the requirement of video packets from the different user devices of the vehicle. The proxy 
includes a buffering module that manages the video streams. Video transmission is carried out using the DASH standard, briefly reviewed in the next section.

Users employ their mobile devices to connect to the Wi-Fi network of the vehicle and access live contents, among other available multimedia contents.

\subsection{DASH}

DASH [27] is an ISO (International Organization for Standardization) standard for the transmission of live and on demand content. DASH uses HTTP for data transmission and is based on the segmentation of multimedia files. In DASH, each file is encoded with different qualities which are split into little parts called segments. In order to access multimedia content, clients select the quality of each content segment and this selection can be done even during the playback, adapting quality and bitrate to bandwidth constraints and device capabilities.

In this sense, DASH defines a manifest file, called MPD (Media Presentation Description), which describes the multimedia content, the different qualities and how the content has been split into segments. Segments must start with a key-frame, that is, a complete image, in order to remain independent of the rest. Finally, segments are encoded using the desired format and hosted in a server, usually a web server.

Nevertheless, live streaming differs from on-demand streaming in the sense that content is generated continuously in real-time. In this proposal, media content is obtained from TV/radio services so DASH segments have to be also generated continuously.

\subsection{Proxy}

In this proposal, the proxy installed in the vehicle is in charge of creating and managing the buffers for each configured TV channel. Initially, the proxy receives the MPD with information about video segments, and requests the corresponding DASH segments to the cloud afterwards. Unlike on-demand video transmission, where the MPD is created at production time and remains unchangeable, in live video transmission the MPD is updated continuously as the video packets are encoded and packetized. Thus, the MPD is sent periodically to inform about new available segments and each segment has time stamps. The proxy has the responsibility for creating a new MPD containing the description of segments in the local buffer, not in the cloud, and for adjusting their timestamps accordingly. If any media segment could not be downloaded for any reason, the proxy could ask the cloud server to retransmit that lost segment as long as clients had not started playing it yet. In this sense, the proxy fills the buffer, which is shared by all clients, before allowing final users to request contents. Since the buffering process is carried out at the proxy, only one flow is requested to the cloud, in contrast to the multiple flows that would be fetched if every client requested the flows instead. It is worth noting that the proxy is completely transparent to the clients.

In this approach, video quality is selected when content transcoding is carried out at the cloud and it is not changed later. For simplicity, although DASH standard enables the possibility to send several qualities of the same video, only one quality is sent to the on-board server. In this case, the proxy adjusts the amount of segments in buffer according to the measured bandwidth. The proxy is designed to adjust the amount of segments in buffer with the aim of providing continuous playback in client players on-board. Implementing a quality selection algorithm in our developments would introduce more complexity, since it is a real implementation and not just a simulated study, and we plan to address that in the future. 
In order to let the clients play the content transparently, the generated MPD must contain new timestamps for each segment, time-shifted according to the buffer capacity. Therefore, the timestamp for the segment $i$ buffered in the proxy $t_{\text {proxy }}(i)$ will be:

$$
t_{\text {proxy }}(i)=t_{\text {cloud }}(i)+T_{\text {buffer }}
$$

where $t_{\text {cloud }}(i)$ is the presentation timestamp of the original segment generated in the cloud and $T_{\text {buffer }}$ is the proxy buffer length in seconds. Additionally, $t_{\text {cloud }}(i)$ and $T_{\text {buffer }}$ can be expressed as a function of the segment duration $T_{\text {segment }}(i)$ as follows:

$$
\begin{gathered}
t_{\text {cloud }}(i)=t_{0}+\sum_{j=0}^{i-1} T_{\text {segment }}(j) \\
T_{\text {buffer }}=\sum_{i=j}^{j+N} T_{\text {segment }}(i)
\end{gathered}
$$

where $t_{0}$ is the initial transmission time, and $N$ is the buffer size in segments. Assuming all segments with the same duration $T_{\text {segment }}$, the timestamp of proxy segments can be expressed as:

$$
\begin{gathered}
t_{\text {cloud }}(i)=t_{0}+i * T_{\text {segment }} \\
T_{\text {buffer }}=N * T_{\text {segment }} \\
t_{\text {proxy }}(i)=t_{0}+T_{\text {segment }} *(i+N)
\end{gathered}
$$

As a matter of fact, segment recovery can be implemented attending multiple conditions. Near-deadline segments have usually highest priority, but other strategies could also perform well. For instance, prioritizing single lost segments recovery instead of consecutive segments will prevent users to suffer intermittent interruptions with the downside of having longer ones.

Finally, in case the proxy calculates that the very next lost segment cannot be downloaded before it should be played according to (6), this segment recovery is omitted and loss is assumed. Resources are then employed for downloading or recovering other in-time segments.

\section{Methodology}

The evaluation presented in this paper has been carried out using two different scenarios. On the one hand, assessments were carried out in a laboratory environment, using real TV signal and a DVB-S receiver equipment, transmitting content throughout a Wi-Fi connection and emulating wireless channel losses. On the other hand, a realistic scenario was assessed in order to carry out tests in mobility, capturing DVB-S TV signal on the server side and transmitting it to a mobile vehicle using $4 \mathrm{G}$ connectivity. Following, the methodology used in each one of these two scenarios is described.

\subsection{Simulation environment}

\subsubsection{Testbed}

Fig. 2 presents the testbed used to carry out the evaluation in the laboratory environment.

First of all, TV signal is captured at the cloud server using a DVB-S antenna and a receiver. Then, the server tunes and demultiplexes the configured channels and starts encoding the flows. The video is encoded using H.264 at $500 \mathrm{kbps}$. DASH packetized segments are available to be requested by the proxy connected to the access point. So far, these elements involve the cloud side of the architecture. 
It is worth mentioning that video quality is selected when content transcoding is carried out at the cloud and it is not changed later. As aforementioned, only one quality is sent to the on-board server. In this case, the proxy adjusts the amount of segments in buffer according to the measured bandwidth. The proxy is designed to adjust the amount of segments in buffer with the aim of providing continuous playback in client players on-board.

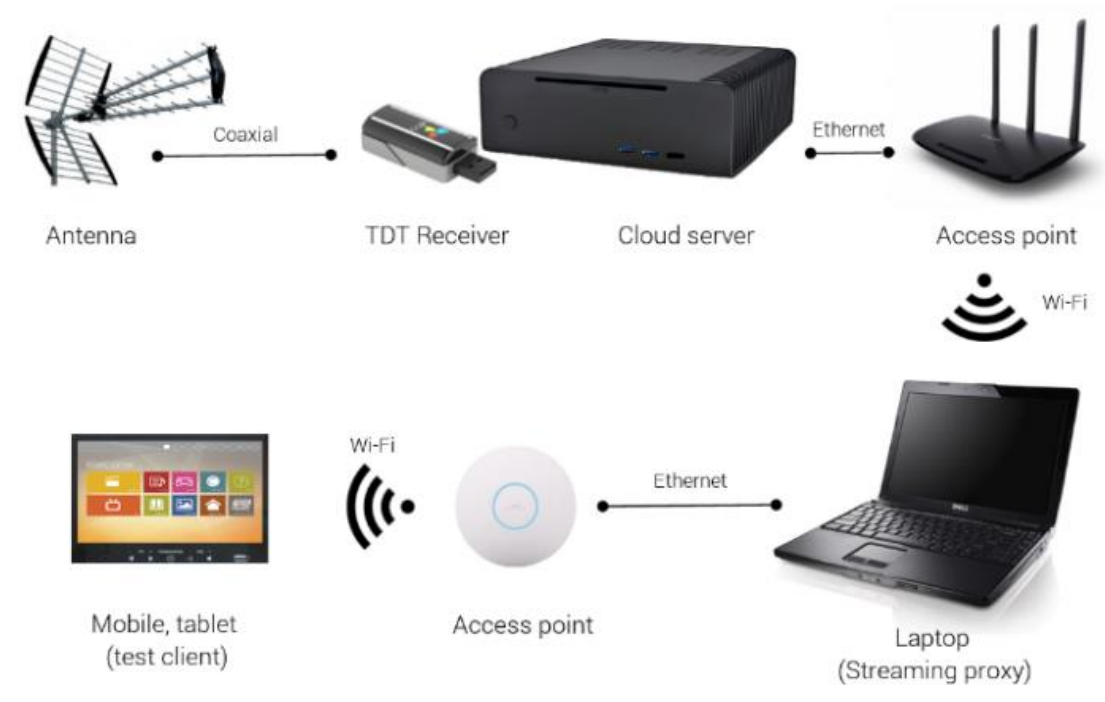

Fig. 2. Laboratory testbed

Therefore, the proxy service runs on a laptop connected to the access point. This Wi-Fi channel will be tweaked to emulate losses, delay and bandwidth constraints with the aim of assessing the system response in non-ideal situations and anticipating the behavior when using $4 \mathrm{G}$ connectivity in mobility. The proxy will be responsible for downloading segments from the cloud, filling the local buffer and streaming the content when requested.

In this sense, once the proxy manages to buffer video segments, these will be set available to final clients through the local access point. The test clients are mobile devices, such as smartphones or tablets.

\subsubsection{Evaluation parameters}

In this environment, the main evaluation parameter is the state of the buffer on the reception player. This buffer size has been set to 3 segments (i.e. 30 seconds given a segment duration of 10 seconds). However, the buffer level can reach higher levels if the current playing segment is also taken into account. Moreover, the buffer level is closely related to the user perception. Hence, when the player buffer is completely empty, the user perceives video interruptions, and therefore, a considerably degradation in the Quality of Experience.

For the evaluation, different scenarios are presented where packet delay, losses and bandwidth vary during 3 minutes. These scenarios have been selected in order to simulate the most likely scenarios in a real environment. The proxy buffer in simulated scenarios is set to 70 seconds, i.e. 7 segments of 10 seconds.

In addition, Quality of Experience of final users has been evaluated using the ITU-T P.1203 recommendation [28]. This recommendation describes a set of objective parametric quality assessment modules that help to predict the impact of media encoding and observed IP network impairments on quality experienced by the end-user in multimedia streaming applications. The recommendation includes four different modes of operation, with different complexity both of the input information and the model algorithms. In this paper, we use the most complex of the modes, the mode 3. This mode may access up to overall media stream information (based on bitstream parsing), 
apart from having access to codec information, target bitrate, frame rate, segment duration, coding and display resolution (as mode 0) and access to video frame sizes and durations, and video frame types (as mode 1) [29].

\subsection{On-board environment}

\subsubsection{Testbed}

The on-board environment represents a more realistic scenario. This testbed uses a dedicated server for TV capturing on the cloud side and a car-powered server to be carried in the vehicle and perform the proxy tasks.

The cloud server was installed in the headquarters of a collaborating partner. The cloud server is in charge of receiving the TV/radio signal, processing the channels and managing the requests from the shipped server in the vehicle. As in the simulation scenario, the bitrate of the video has been set to $500 \mathrm{kbps}$ using H.264. This video rate is used by the service provider since it provides a good trade-off between the use of the 4G channel (and the associated costs) and the Quality of Experience perceived by the users. To that extent, different subjective studies (not included in this paper) were carried out to validate the Quality of Experience of users under different video rates and reception devices with different screen sizes. Fig. 3 shows the cloud server, which receives the DVB signal through a satellite antenna and encodes the flows.

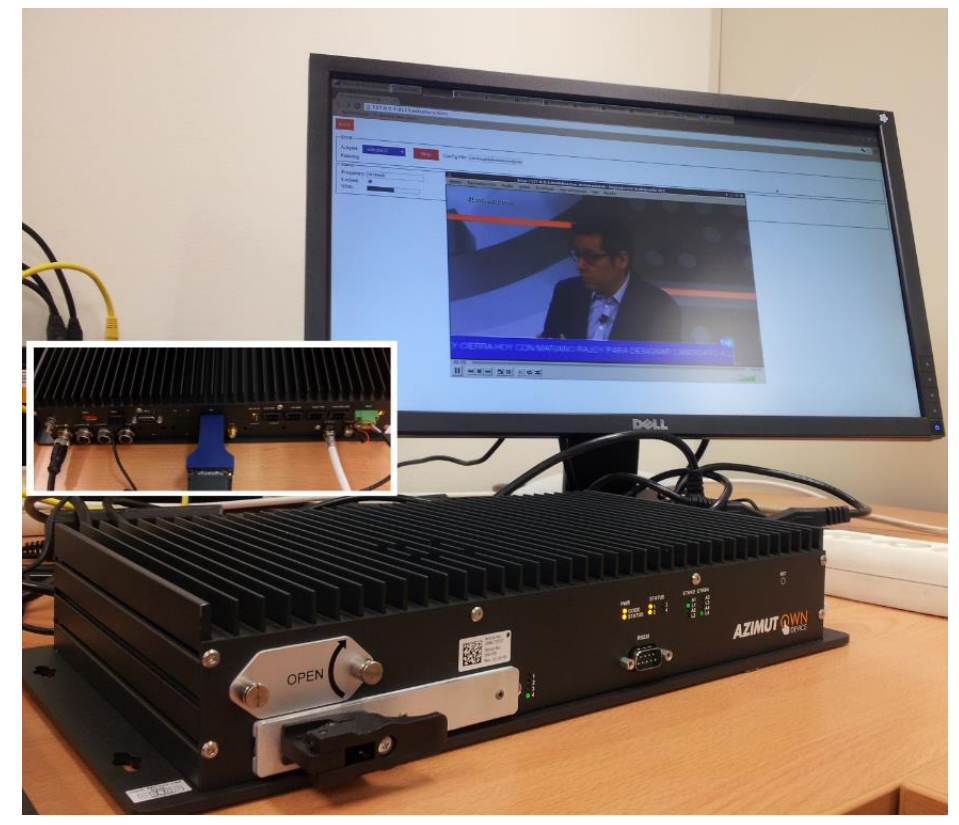

Fig. 3. Cloud server equipment

As aforementioned, in order to carry out mobility tests, a shipped server was installed in a vehicle (in this case, a car). The on-board environment has four different parts: server, $4 \mathrm{G}$ router, $4 \mathrm{G}$ antenna and one or more access points. This could seem overwhelming if we intend to install the system in a car as is, but there are some aspects to take into account. On the one hand, the system is designed for bigger vehicles, such as buses or ferries, where some location can be found to place the on-board equipment. On the other hand, the on-board system can be implemented in one single equipment. Nowadays, there are smart Wi-Fi access points with $4 \mathrm{G}$ connectivity that can be used instead. The main drawback is the processing capabilities and the difficulty to access the operating system in order to implement the proxy mechanism.

With this trade-off in mind, we installed the shipped system in a car to perform the assessment, as shown in Fig. 4. This server acts as a proxy and is in charge of receiving the TV/radio flow through 4G (using a 4G antenna and 
a $4 \mathrm{G}$ router) and managing requests from the final clients. Regarding the client side, a laptop was used to take the measurements and check the buffer level of the live player. Those measurements are presented in the evaluation section and allow the comparison between the proxy performance and the direct live transmission through $4 \mathrm{G}$ (without proxy). The laptop is connected to the proxy by means of an access point also installed in the car. Fig. 4 depicts the set-up used to carry out the tests. The $4 \mathrm{G}$ router also stores geolocation information, which will also be used in the presented evaluation.

Precisely, from the information obtained from the 4G router regarding the GPS measurements, the itinerary taken by the vehicle to perform the tests has been marked out in Fig. 5. The total duration of the tests was 1450 seconds (24 minutes approximately) for a trip of about 30 kilometers. In the figure, three clearly differentiated sections (marked in different colors) can be seen. Each one of these three sections is analyzed separately in the evaluation section: section 1 (in blue color), section 2 (purple) and section 3 (orange). The itinerary alternates rural areas (mainly secondary roads with poor connectivity) and urban areas (where there is good connectivity). In this way, it has been possible to evaluate the performance of the proxy in different environments, especially when packet losses occur.

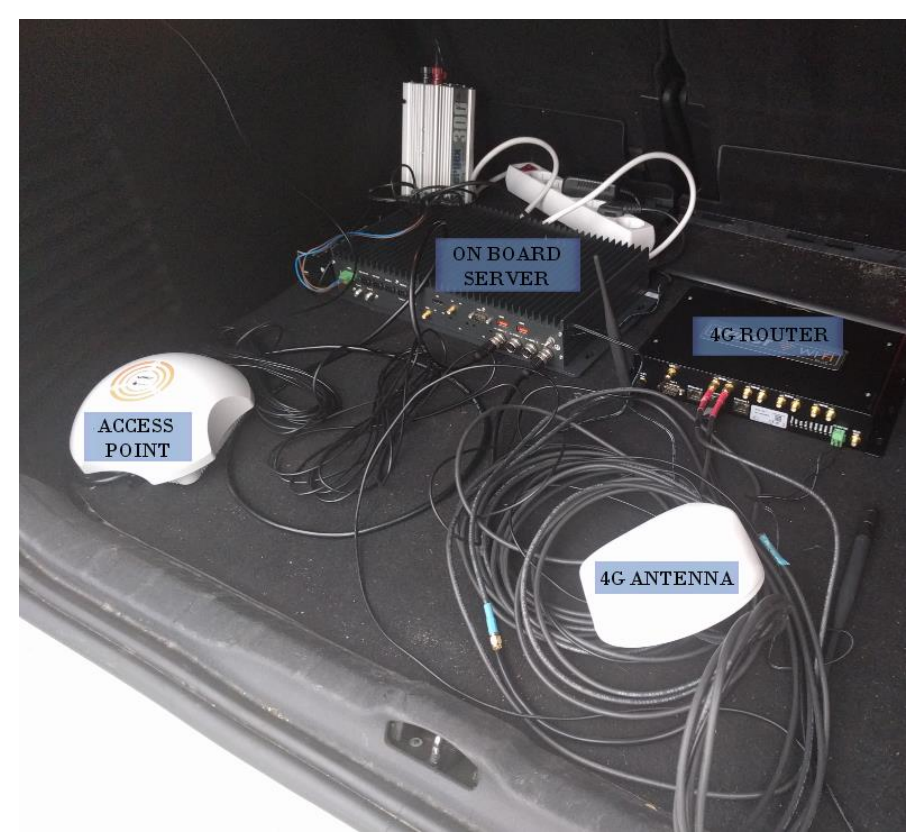

Fig. 4. Assembly of the system in the vehicle

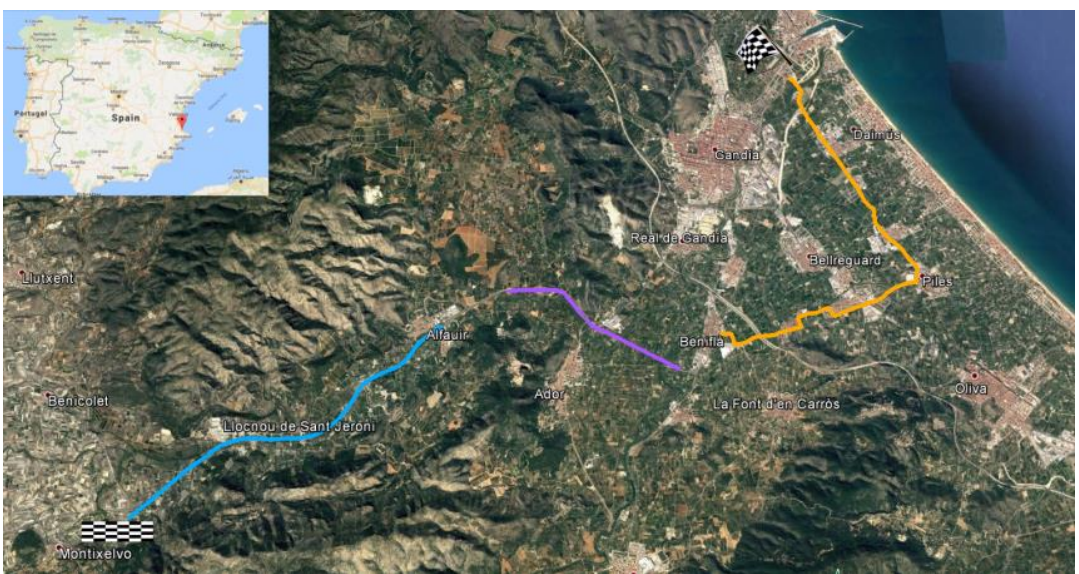

Fig. 5. Itinerary followed to perform the tests 
Regarding the proxy, the proxy buffer in the on-board environment has been set to 150 seconds to cover the expected interruptions due to lack of coverage or hand overs. In addition, the player buffer has been configured with 30 seconds.

\subsubsection{Evaluation parameters}

Again, the main evaluation parameter is the player buffer level, considering factors as the bandwidth, packet losses and delay. In addition, the number and duration of playback interruptions will be analyzed since they have a decisive impact on the user experience. Also, the recommendation ITU-T P.1203 [28] is used to evaluate the Quality of Experience of final users as explained in Section 4.1.2, and specifically, mode 3 of this recommendation.

\section{Evaluation}

\subsection{Simulation environment}

As aforementioned, there are eight different scenarios under consideration. In those scenarios, losses, delay and bandwidth have been analyzed. Each study is presented in the following sections:

\subsubsection{Bandwidth}

Regarding bandwidth constraints, four different channels have been simulated with different instant available bandwidth. The first channel profile is shown in Fig. 6a in dotted green color. The bandwidth varies repeatedly between $100 \mathrm{Mbps}$ and $0 \mathrm{Mbps}$, simulating moments of maximum connectivity and moments of no connectivity at all.

Also, Fig. 6a shows the state of the player buffer when the playback is carried out using the on-board proxy and when it is not, i.e. direct playback from the cloud server. Results show that the use of the proxy allows the buffer level to remain between 10 and 17 seconds, regardless the available bandwidth of the $4 \mathrm{G}$ link. In contrast, in live transmission the buffer presents higher fluctuation. Thus, although reaching levels of 29 seconds at certain points, it drops at some intervals and all buffered video is consumed. This situation is not desirable since users are highly likely to perceive interruptions in the video reproduction when the buffer is completely empty. Comparing buffer levels with the bandwidth profile, some conclusions can be inferred. When the bandwidth is high, the buffer of live transmission increases considerably, because each time there is enough bandwidth the player downloads as much content as possible until filling the internal buffer. On the contrary, each time an interruption occurs the player stops, resuming the reproduction when there are enough video segments in the buffer. This causes a delay on the video reproduction. At the end of the 3-minute test, a total delay of 12 seconds $(6.6 \%$ of the duration of the test) is perceived using the live player. It is worth noting that the service interruptions have to last at least 30 seconds to make the buffer empty completely.

On the other hand, the proxy buffer length is higher than the duration of the interruptions, so the proxy has been able to absorb the losses and recover the segments during the intervals of maximum coverage, keeping the player buffer at a healthy level and allowing a continuous playback. In this way, in this first scenario, significant improvements can be seen when using the proxy. These improvements will remain as long as the proxy buffer is higher than the maximum duration of the moments with no coverage and there is enough bandwidth to recover the lost segments during the moments with coverage. Therefore, an appropriate buffer dimensioning is essential to prevent the buffer from resulting empty. 
The second assessed channel simulates a hand over situation where coverage is lost for 1 minute. Fig. $6 \mathrm{~b}$ depicts the bandwidth variation and the buffer level for live and proxied transmission. When the receiver is within the coverage area, the bandwidth reaches $3 \mathrm{Mbps}$, which is in tune with real mobile technologies like 4G. The existence of such a long region without reception has a significant impact on the state of the buffer, especially on live transmission, where there is a section with a duration higher than 50 seconds in which the buffer is empty. That is, once the player has consumed the almost 20 seconds initially available in the buffer, it runs out of video segments to play. On the other hand, when using the proxy, the buffer never empties completely, although it drops dramatically. Finally, the live player recovers video segments and manages to fill the buffer, but it ends with a delay of 51 seconds $(28.3 \%)$.

Once again, results show the convenience of using the proxy and, especially, how important a correct configuration of the buffer size to avoid coverage cuts is. Comparing these results with those obtained in channel 1 , it can be noted that less frequent but longer interruptions are a higher threat for a seamless video reproduction and QoE if the buffer of the proxy is not dimensioned appropriately.

Regarding channels 3 and 4, the bandwidth distribution and the analysis of the buffer using live transmission and proxy (shown in Fig. 6c and Fig. 6d, respectively) lead to the same conclusions as in previous channels. That is, the use of the proxy helps the player maintain a steady buffer level, thus minimizing the number of interruptions.

\subsubsection{Delay}

This section presents the tests carried out to evaluate the packet delay and the effects it has on the buffer level. For this purpose, two different types of channels have been considered. The delay distribution and the resultant buffer level for channel 5 and channel 6 are shown in Fig. 6e and Fig. 6f, respectively. Note the different axis scale for delay in both scenarios. The first scenario entails two zones of high delay whereas the second one describes high variance with low and moderate packet delay.

Regarding channel 5, Fig. 6e points out that high delay values lead the buffer to empty in live transmission. When the delay decreases, the buffer increases considerably, reaching values of 30 seconds. On the contrary, as in the previous studies, the use of the proxy guarantees a steady buffer. Playback interruptions and the process of filling the buffer in the live player result in a delay of 32 seconds (12.7\%) taking into account the whole playback length.

Results for channel 6 are depicted in Fig. 6f. Since the average delay is lower than in the previous case, the length of interruptions is also much lower. In fact, there are few interruptions in live transmission, and the duration is practically lower than 5 seconds. Comparing the transmission using the proxy with that for live transmission, once again the behavior of the buffer in the proxy is steadier than live transmission, reducing the probability of a buffer underrun.

\subsubsection{Losses}

In this section, the amount of losses has been changed over time to create the channels to assess. On the one hand, channel 7 presents three zones of high packet loss and zones without losses (Fig. 6g). On the other hand, channel 8 has lower packet losses but they are presented continuously (Fig. 6h).

Regarding the analysis of channel 7, a channel with these loss peaks implies interruptions in video transmission, especially in live transmission, as depicted in Fig. 6g. The final delay introduced during the simulation using the live player is 27 seconds (a $15 \%$ of the total time of simulation). On the other hand, in the proxy-based transmission architecture, the buffer level remains rather stable, and the buffer never results empty. 
With respect to channel 8, Fig. 6h shows that although maximum losses are lower than in the previous test, the live player presents some video interruptions. This is due to the fact that the continuous packet loss prevents the buffer from receiving new full segments. Comparing with the proxy-based player, live player has higher variability and risk of completely running out of video segments in the buffer.

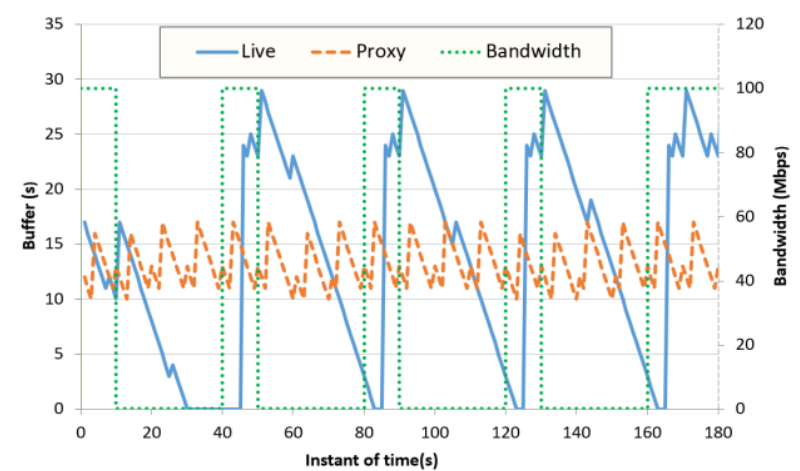

a) Channel 1: periodic bandwidth

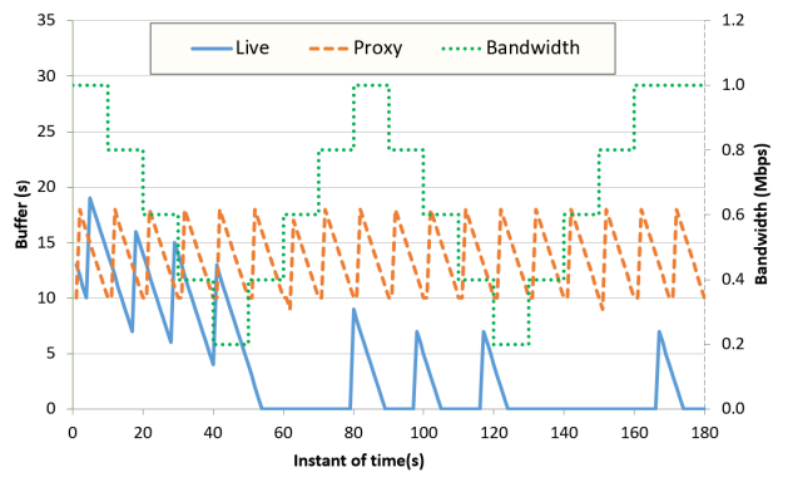

c) Channel 3: stepped bandwidth

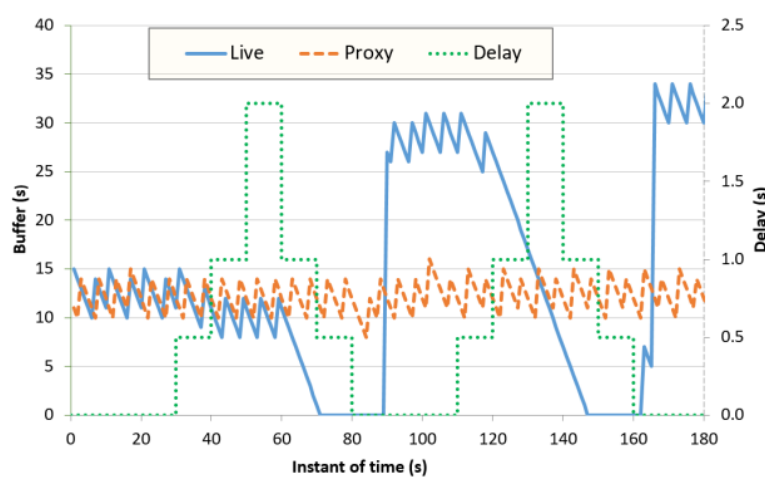

e) Channel 5: stepped delay

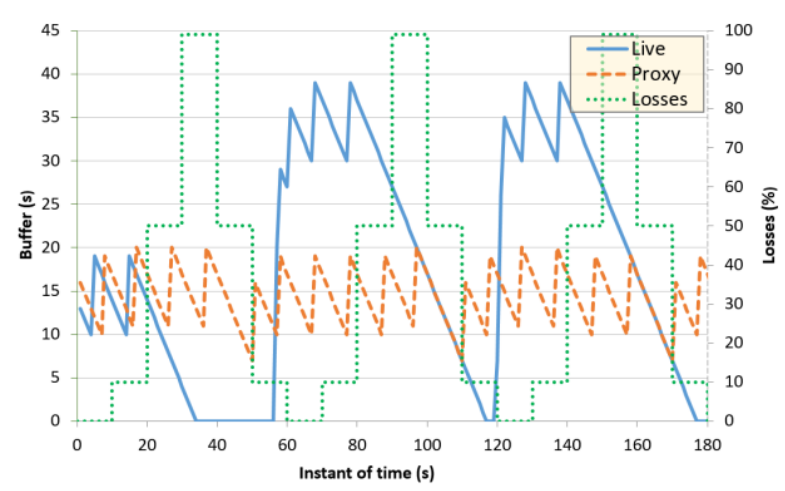

g) Channel 7: stepped losses

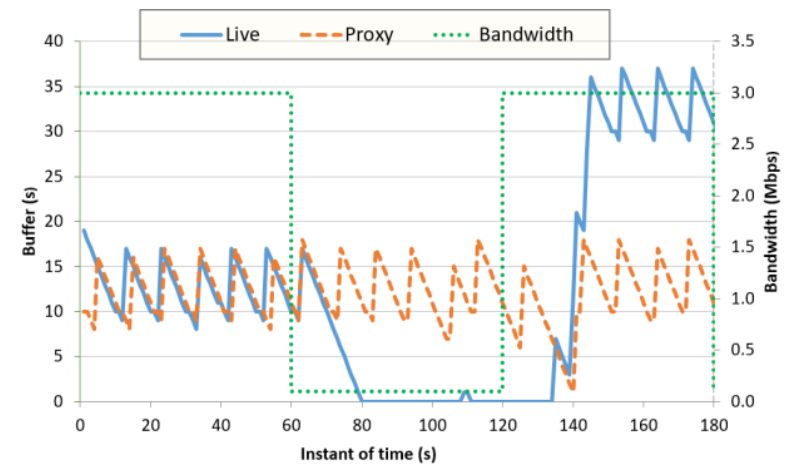

b) Channel 2: long fall of bandwidth

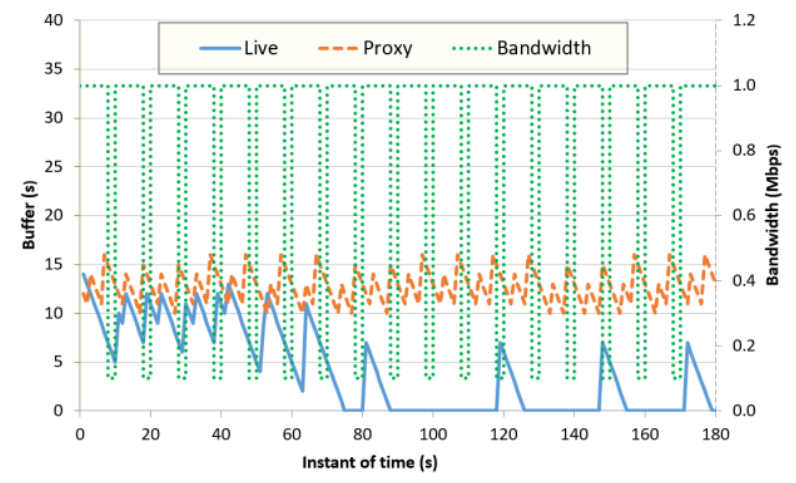

d) Channel 4: brief fall of bandwidth

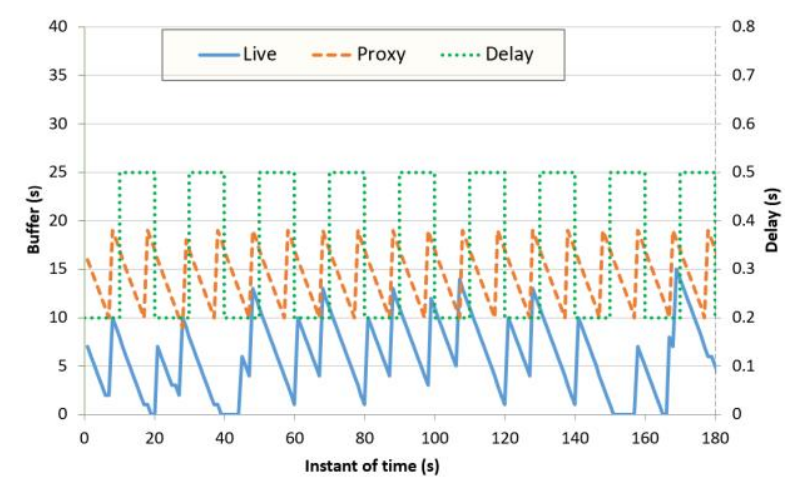

f) Channel 6: periodic delay

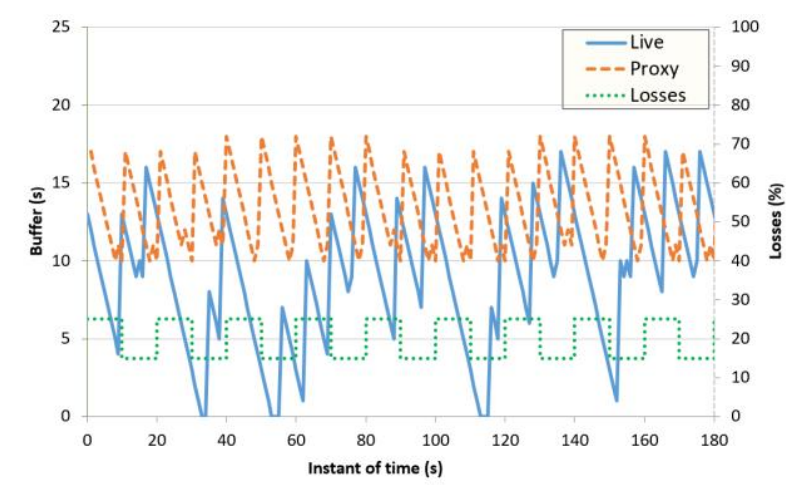

h) Channel 8: periodic losses

Fig. 6. Bandwidth and buffer evaluation 


\subsubsection{MOS Evaluation}

One of the most used techniques to measure QoE is the Mean Opinion Score (MOS), in which different users value their experience regarding a video playback analyzing certain parameters using a scale between 1 (lowest satisfaction) and 5 (highest satisfaction) [30]. Table 1 shows the MOS evaluation carried out using the recommendation ITU-T P.1203 for live and proxy playback in the eight simulation channels. The same video (which duration is 180 seconds and its bitrate is $500 \mathrm{kbps}$ ) has been used in all scenarios. For this reason, regarding the proxy, as no stalls occur, all the channels have the same MOS (4.29), a rather good value, taking into account that the MOS varies from 1 (worst experience) to 5 (best experience). On the contrary, mainly because of the existence of stalls, live transmission provides much worse values of MOS (between 2.35 and 3.24). According to the table, the worst scenario is Channel 3. This is in line with Fig. 6c, which shows that this is the channel with the largest number of stalls and duration.

Table 1. MOS evaluation using ITU-T P.1203

\begin{tabular}{|l|r|r|r|r|r|r|r|r|}
\cline { 2 - 9 } \multicolumn{1}{c|}{} & Channel 1 & Channel 2 & Channel 3 & Channel 4 & Channel 5 & Channel 6 & Channel 7 & Channel 8 \\
\hline Live & 2.84 & 3.24 & 2.35 & 2.48 & 3.11 & 2.88 & 2.80 & 3.24 \\
\hline Proxy & 4.29 & 4.29 & 4.29 & 4.29 & 4.29 & 4.29 & 4.29 & 4.29 \\
\hline
\end{tabular}

\subsection{On-board environment}

\subsubsection{Losses, delay and buffer level}

Although simulated channels have been very useful to evaluate how players behave with and without the developed proxy, the assessment in real scenarios is a key factor to determine the feasibility of the proposed architecture. However, one of the drawbacks of real environments is the difficulty to define the channel during the experiment. Therefore, during the on-board test, the $4 \mathrm{G}$ link losses have been continuously measured, as well as the packet delay, in order to model the channel. These measurements are shown in Fig. 7 and Fig. 8, which depict the link quality in terms of packet losses and delay, respectively.

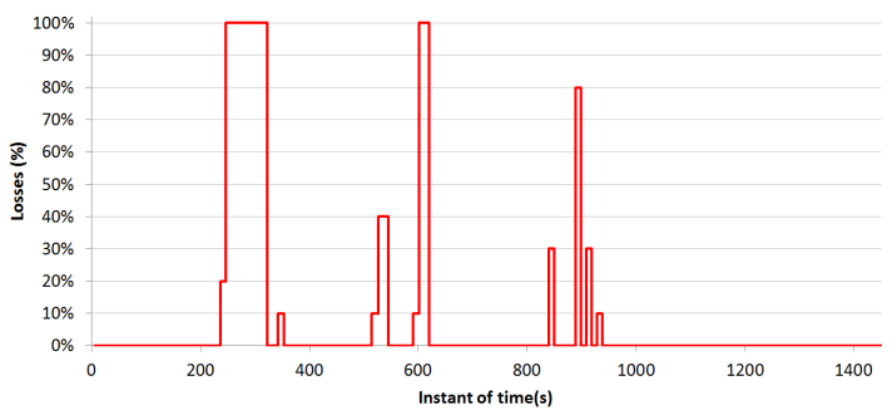

Fig. 7. Losses of the $4 \mathrm{G}$ link

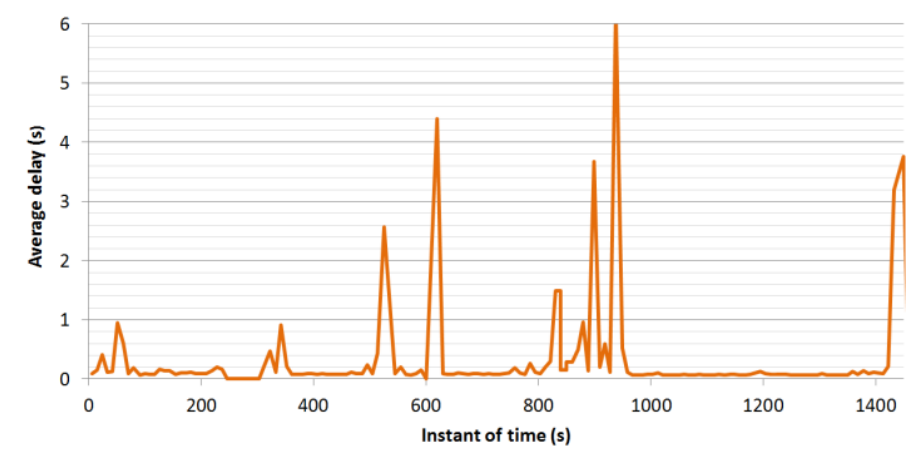

Fig. 8. Average packet delay of the $4 \mathrm{G}$ link 
In Fig. 7 three zones where packet losses reach a considerable magnitude can be distinguished. This has been very useful to evaluate the effectiveness of the buffering technique on the shipped server, which uses the proxy. About the measured delay, Fig. 8 shows that the maximum delay is 6 seconds, and comparing the results shown in Fig. 7 and Fig. 8, a correlation between losses and delay can be inferred.

In order to thoroughly analyze the areas where there is a significant deterioration in the $4 \mathrm{G}$ link quality, the route has been divided into 3 sections, as drawn in Fig. 5. These sections of interest are highlighted in Fig. 9, where the buffer level for both live and proxy-based transmission is depicted. Outside these areas, video reception was paused to perform operation and maintenance tasks, so no measurements were taken. It is worth remembering that the maximum capacity of the player buffer has been set to 30 seconds. In order to throw neater results, each section is analyzed independently.

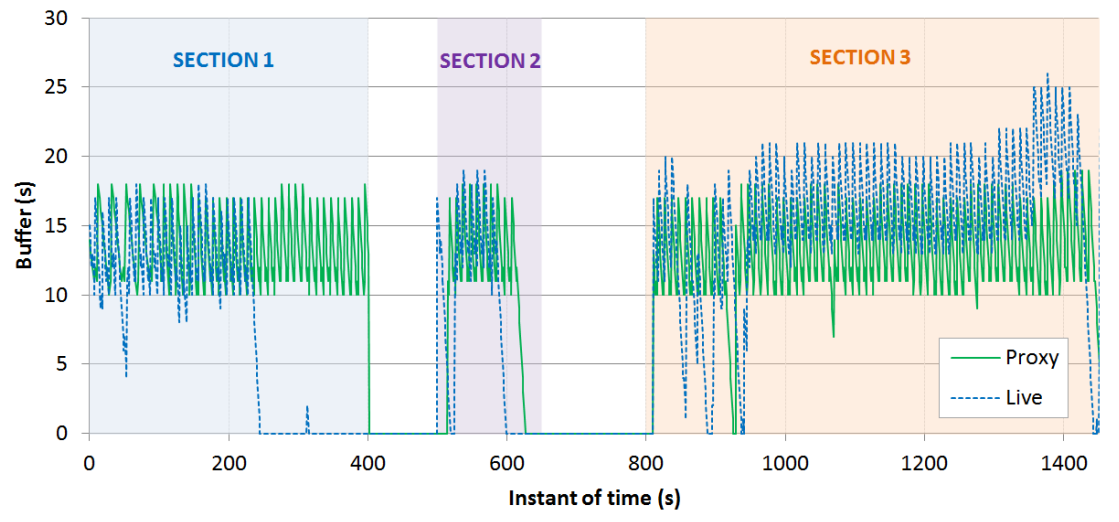

Fig. 9. Buffer evaluation in on-board scenario

The first section, which is drawn in blue color in Fig. 5, comprises the time from 100 to 400 seconds. Fig. 10a shows the link quality regarding the packet loss measured in this interval of time. As the figure depicts, the signal is lost during more than 1 minute. The effect that these losses have over the visualization of the content in a player that accesses directly to the cloud (live) and another that obtains the data from the proxy, can be seen in Fig. $10 \mathrm{~b}$.

From these results, it can be verified that when channel losses begin to increase (at around 240 seconds) live reproduction exhausts the player buffer, thus causing interruptions in the visualization and the consequent deterioration of QoE. In this experiment, although packet losses decrease, live player fails to refill the buffer because the high packet delay. In contrast, by using the proxy, the buffer is kept within a safe range while waiting for the connectivity to return. As during the tests the proxy has been set with a buffer of 150 seconds, this has been enough time for the vehicle to come back to an area with sufficient connectivity and to download the following video segments needed to fill the buffer. The result has been a continuous reproduction during all the section using the player through the developed proxy.

The second section comprises the time from 520 seconds to 625 seconds (purple color in Fig. 5). The losses level as well as the average delay of section 2 can be seen in Fig. 11a. There are two main loss areas, one with $40 \%$ of packet losses and another with a complete loss of connectivity. Those losses affect both the direct connection and the proxy-based one, as Fig. 11b shows.

Results show that the first loss area does not affect neither the live connection nor the proxy, because the duration is shorter than the player buffer and there are always video segments ready to be played. The second loss area affects both live and proxy-based connection. Although the proxy buffer length could absorb both zones with losses separately, the closeness of zones with high loss rate together with the increase of packet delay prevent the 
player buffer from requesting lost segments and, therefore, the video playback results interrupted eventually, particularly over $625 \mathrm{~s}$.

The last section, section 3, is the largest one, with a time interval between 800 and 1450 seconds (more than 10 minutes). Due to its different behavior regarding coverage, this section has been divided into two different areas:

- Section 3a, which is a critical area because the connectivity is intermittent. This area runs through secondary roads.

- Section $3 b$, where the connectivity is excellent, without coverage losses. This is in line with the fact that coastal zones usually have good connectivity because population is higher.

The distribution of losses in section 3 is shown in Fig. 12a, which reflects the two sub-sections clearly differentiated. In Section 3a, between 800 and 1000 seconds, packet losses and delay are significant. However, Section $3 b$ presents total connectivity for more than 5 minutes. Additionally, the buffer level can be seen in Fig. $12 \mathrm{~b}$.

To see in more detail the behavior in the critical area, Fig. 13a shows the losses level and the average delay for Section $3 \mathrm{a}$ and Fig. 13b illustrates the buffer level. In this case, transmission presents peaks of high losses and delay, which lead to empty the player buffer in both live and proxy-based connection at some points. Nevertheless, interruptions using live connection are longer and more frequent than those produced in the proxy-based connection. In this case, without taking into consideration the initial warm-up zone, the player buffer empties for 4 seconds, whereas in the direct connection there are 3 interruptions with a total duration of 10 seconds.

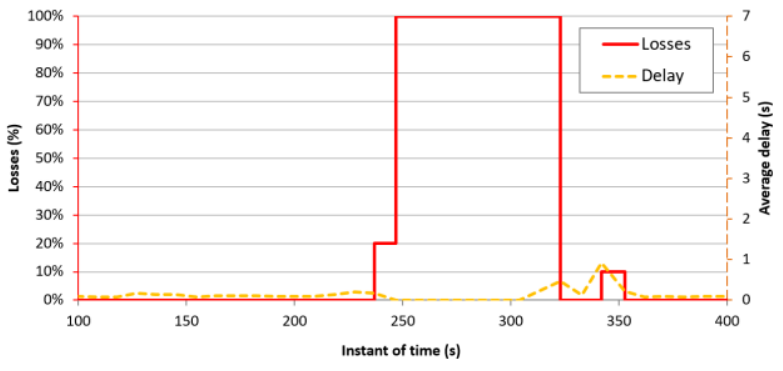

a) Losses level and average delay

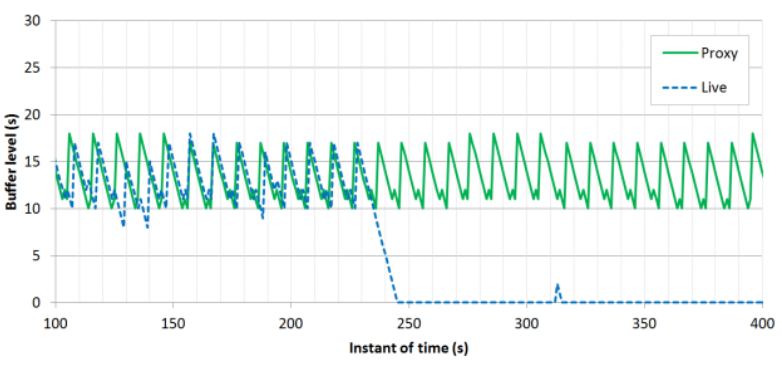

b) Buffer level

Fig. 10. Section 1

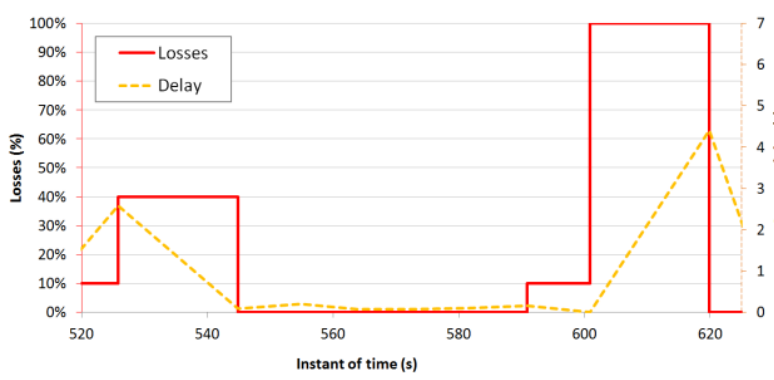

a) Losses level and average delay

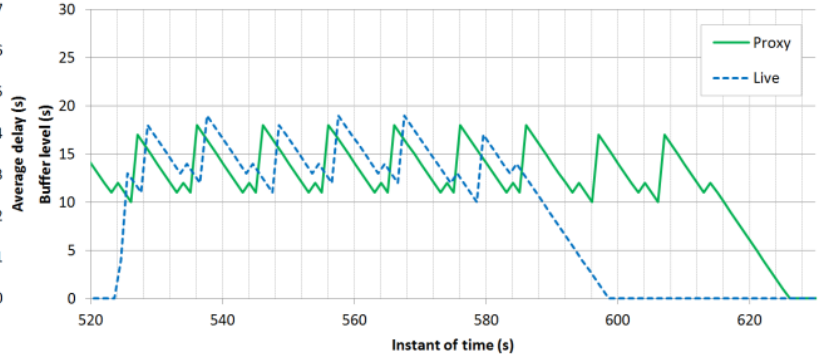

b) Buffer level

Fig. 11. Section 2 


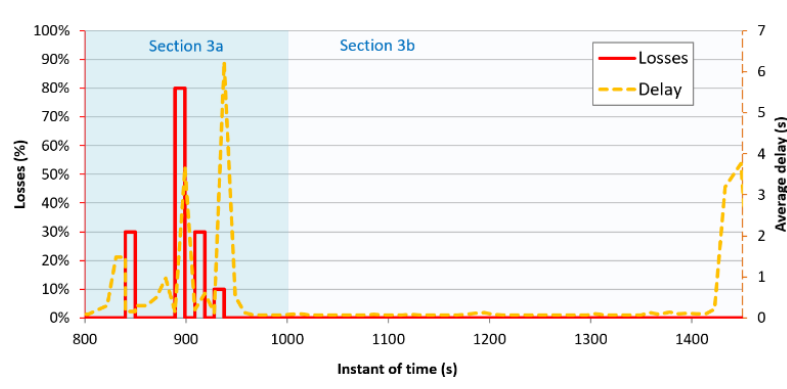

a) Losses level and average delay

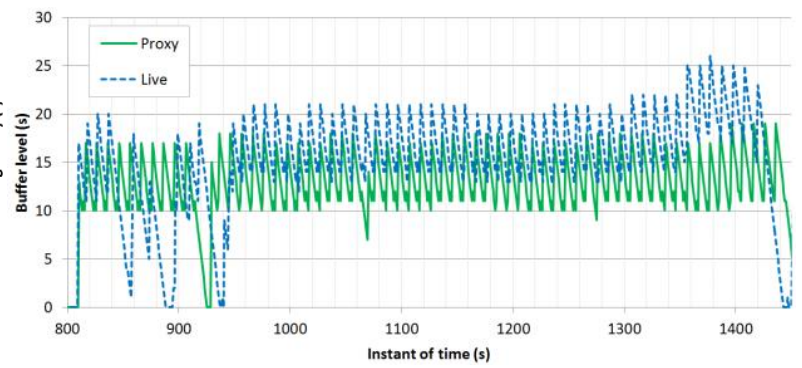

b) Buffer level

Fig. 12. Section 3

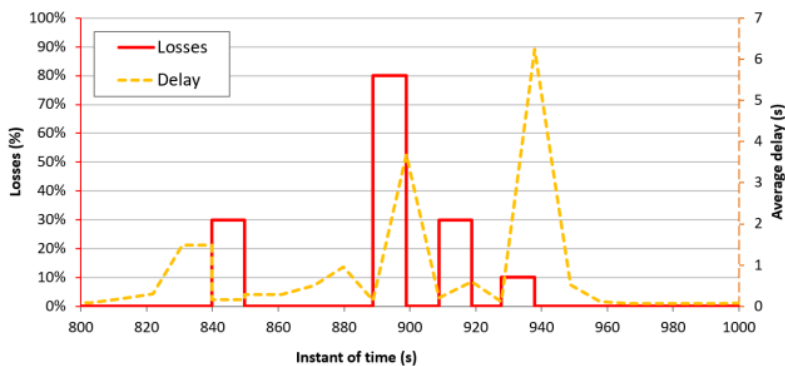

a) Losses level and average delay

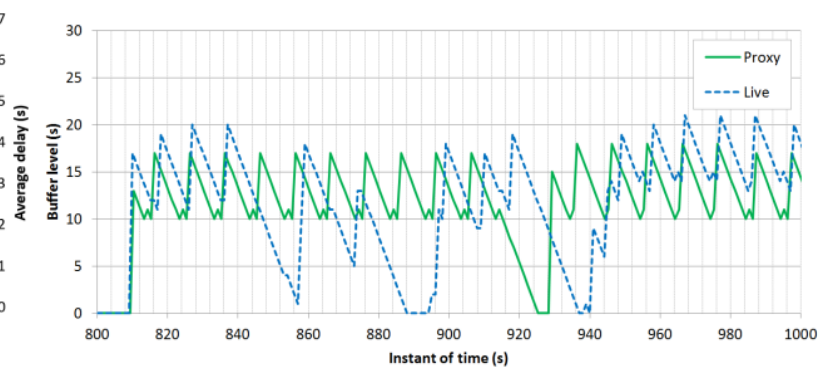

b) Buffer level

Fig. 13. Section 3a (critical area)

\subsubsection{Interruptions}

After having evaluated the behavior of the developed system in different real mobility scenarios, it is interesting to quantify the number (and duration) of interruptions (stalls) to be able to estimate the level of improvement introduced.

Thus, Fig. 14 shows the accumulated time during which the players have interrupted their reproduction because their internal buffer empties. As can be seen, each section has been analyzed separately to have different intrinsic characteristics in terms of signal level and coverage. Finally, the total number of interruptions during the overall trip is shown.

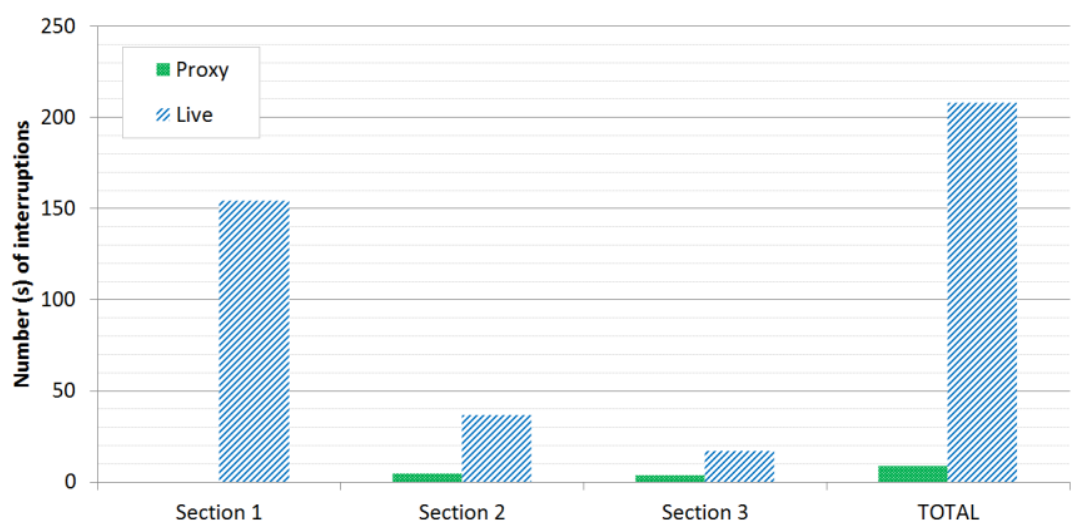

Fig. 14. Duration of interruptions (s)

It is worth noting that it is not possible to compare the number of interruptions in absolute value between one section and another, since each section has a different duration. So, it is more relevant to compare the percentage of time where the reproduction has been interrupted. The values of the duration of the section and the number of stalls are shown in Table 2, both per section and as a whole. 
Table 2. Number and percentage of stalls

\begin{tabular}{|c|c|c|c|c|c|c|}
\hline & & \\
\hline & & Sec. 1 & Sec. 2 & Sec. 3 & Sec. 3a & Total \\
\hline \multicolumn{2}{|c|}{ Duration (s) } & 301 & 111 & 641 & 191 & 1053 \\
\hline \multirow{3}{*}{ Proxy } & Number of stalls & 0 & 1 & 1 & 1 & 2 \\
\hline & Stalls duration (s) & 0 & 5 & 4 & 4 & 9 \\
\hline & $\%$ & $0.0 \%$ & $4.5 \%$ & $0.6 \%$ & $2.1 \%$ & $0.9 \%$ \\
\hline \multirow{3}{*}{ Live } & Number of stalls & 1 & 2 & 3 & 2 & 6 \\
\hline & Stalls duration (s) & 154 & 37 & 17 & 10 & 208 \\
\hline & $\%$ & $51.2 \%$ & $33.3 \%$ & $2.7 \%$ & $5.2 \%$ & $19.8 \%$ \\
\hline
\end{tabular}

For section 1, results from the table show that the long interruption the transmission has suffered has caused the live player to empty the buffer for a long period of time (51.2\%). In contrast, the use of the proxy in section 1 has allowed to maintain a continuous video reproduction because the buffer size is larger than the duration of the gap without coverage.

Regarding section 2, the table depicts that the reception is interrupted during the $33.3 \%$ of time for live transmission, whereas the proxy keeps on mitigating many channel losses, interrupting the reproduction for only the $4.5 \%$ of the time. This is due to the interval with high losses and, especially, to the high packet delay suffered in this interval. In the same line, the critical area of section 3 shows that the reproduction using the proxy presents some moments in which the player is not able to fill the buffer at the required rate, although only the $2.1 \%$ of time against $5.2 \%$ of the live reproduction.

If we take the route globally, there have been interruptions during the $19.75 \%$ of time using live reproduction against only $0.85 \%$ using the developed proxy, which implies an improvement of $95.7 \%$.

\subsubsection{MOS Evaluation}

Table 3 shows the calculated MOS using ITU-T P.1203 for the on-board scenario. We can see that, as expected, the proxy outperforms the live transmission in terms of MOS in the three sections considered. Comparing Table 2 and Table 3, we check that results are coherent, since Section 1 and Section 2 are the sections with the highest percentage of stalls in the live scenario, so both sections have the lowest values of MOS. It is remarkable that, in the proxy scenario, despite having stalls, Section 3 provides better values of MOS than Section 1 (where no stalls occur). In this sense, it is important to indicate that many factors affect the MOS calculated using the ITU-T P.1203, such as stalls, video quality, size of the screen, etc. In this case, the particular characteristics of the video (luminance, movement...) received in that moment in the bus in motion are responsible for the difference between MOS values. In any case, it is worth highlighting that, although the video received in each one of the three sections is different, when evaluating the live and the proxy scenario in each section, the video used is the same, so the comparison presented is valid.

Table 3. MOS evaluation using ITU-T P.1203

\begin{tabular}{|l|r|r|r|r|}
\cline { 2 - 5 } \multicolumn{1}{c|}{} & Section 1 & Section 2 & Section 3 & Section 3a \\
\hline Live & 2.77 & 2.98 & 3.47 & 3.23 \\
\hline Proxy & 3.47 & 3.43 & 3.81 & 3.51 \\
\hline
\end{tabular}




\section{Proxy improvements}

As mentioned, the proxy is in charge of creating and managing the buffers associated to each video flow offered in the platform. Thus, the proxy is designed to adjust the amount of segments in buffer with the aim of providing continuous playback in on-board clients. In the on-board environment presented in this paper, due to commercial requirements, the proxy has basic functionalities, working as a kind of advanced buffer. Nonetheless, it is important to highlight that a proxy is not the same as a buffer. In this sense, the next table summarizes the main characteristics of a proxy and a buffer.

Table 4. Characteristics of a proxy and a buffer

\begin{tabular}{|l|c|c|}
\hline \multicolumn{1}{|c|}{ Feature } & Proxy & Buffer \\
\hline Segment management (fetching, storage, removal) & Yes & Yes \\
\hline MPD modification and adaptation & Yes & No \\
\hline Segment timestamp modification & Yes & No \\
\hline Smart recovering mechanisms & Yes & No \\
\hline Segment processing (re-encoding, forward error correction techniques) & Yes & No \\
\hline
\end{tabular}

Therefore, the functionalities of the proxy used in the system can be extended, as described below. The current proxy receives DASH video packages for each channel, as well as the MPD associated to each flow. In the transmission of live video, the MPD is generated progressively as soon as video packages are recoded, and therefore it is sent periodically to inform about new segments. If any of the video segments is lost, the proxy can request that segment to the cloud server again. Also, the proxy is in charge of providing both video packets and the MPD to each one of the clients that request it. This MPD will not correspond exactly with the same MPD that the proxy receives, but it is an MPD modified by the proxy to mitigate the possible losses of segments that can be produced in a transparent way to the end user and it has updated timestamps to let client devices play the video stream seamlessly. In order to explain carefully the buffering process, Fig. 15 shows the flow chart of the process carried out in the proxy installed on-board.

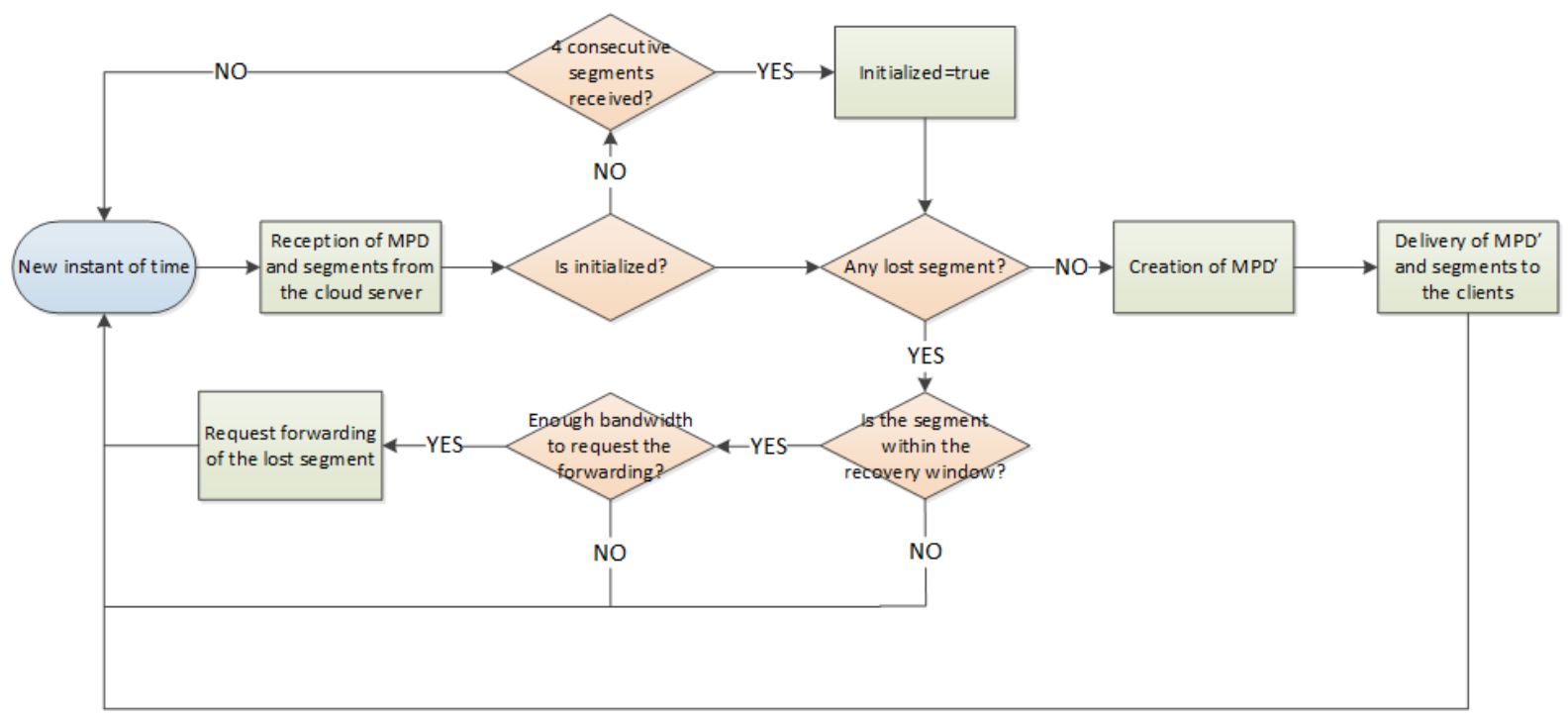

Fig. 15. Flow chart of the proxy functionality 
To fill the buffer existing in the proxy, the proxy first receives the MPD from the video stream so as to reorder the video segments. Then, the proxy tries to download video segments until filling the buffer. Fig. 16 shows an example of proxy operation with a buffer of 12 segments. Each time the proxy receives the MPD it generates an MPD', which will be sent to the on-board clients. The MPD' is a reduced version of the MPD, since it contains information of fewer segments. The clients have a buffer of a certain capacity, for example 30 seconds, so in that case the MPD' will contain information of 4 segments (considering that the segment size is 10 seconds) in order to ensure that client buffers can be filled. Following the example, initially, the MPD' will not be sent until there are 4 consecutive segments available in the buffer (as shown with the dark blocks in Fig. 16a). To prevent clients from connecting and starting to perceive stalls in the reception, the proxy will not send the MPD' to the clients until it has at least 4 consecutive segments (or the configured amount of segments). Clients have a smaller buffer than the proxy because clients cannot request lost packets to the proxy beyond the common operation of the player. On the contrary, the proxy can request lost packets to the server in the cloud as long as there is enough bandwidth that does not worsen the reception of other packets and that allows the segments to arrive in time, so that the proxy will be able to send these video segments to the client devices.

In order to illustrate the advanced proxy operation, Fig. 16a shows an example of the status of the initial buffer of the proxy as it is receiving segments of the server in the cloud. As aforementioned, there are two differentiated areas: the critical area (shown as dark blocks), corresponding to the first 4 segments; and the recovery window (shown as light blocks). In this example, it is considered that the buffer size is 12 video segments and that initially the proxy has received all the segments included in the MPD except segments 2, 5 and 6 (highlighted in red in Fig. 16a). Since the proxy does not have the first 4 segments, it does not generate the MPD' nor send it to the clients. In the following instants of time $\left(t_{2}, t_{3}, t_{4}, t_{5}\right.$ and $\left.t_{6}\right)$ this condition is still not met, so the MPD' is not generated. At the instant $t_{7}$ the proxy already has the first 4 segments, so it generates the MPD' and sends it to the clients, who can start to play live video content.

Following the example shown in Fig. 16b, segment 13, which has not yet been received at the previous instants of time, is finally recovered between the instants of time $t_{8}$ and $t_{9}$. When requesting a segment that has been lost, the proxy first checks if that segment is in the recovery window and, in that case, asks if there is enough bandwidth to request the forwarding of that segment as long as this action does not affect the delivery of other segments. If this condition is not met, i.e. when the available bandwidth does not allow downloading several segments, those segments that are closest to the critical area will have priority. For example, at time $\mathrm{t}_{7}$, fetching segment 13 has more priority than segment 18 .

On the other hand, another recovering priority policy can be applied. If there are many losses in the channel and there are many segments that are not received (as at time $t_{15}$ ), in order not to worsen the Quality of Experience of the users, non-consecutive segments will have preference. For example, at time $t_{15}$, since segments 18 and 19 have been requested during the previous instants and have not been received, and since there are other segments that have been lost and that do not have consecutive order, these non-consecutive segments will have preference (in the example, segments 21, 23 and 25). This is because, in general, it is more annoying for users having many but short stalls than having few although long stalls [31]. Therefore, as the figure shows, segments 18 and 19 cannot be recovered (so that users will perceive a 20 -second video stall, which is understandable in a mobility environment), and segments 21 and 23 will be recovered. 


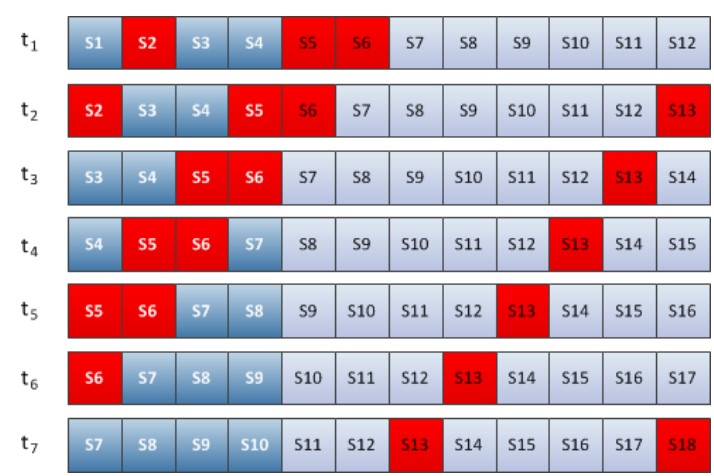

a)

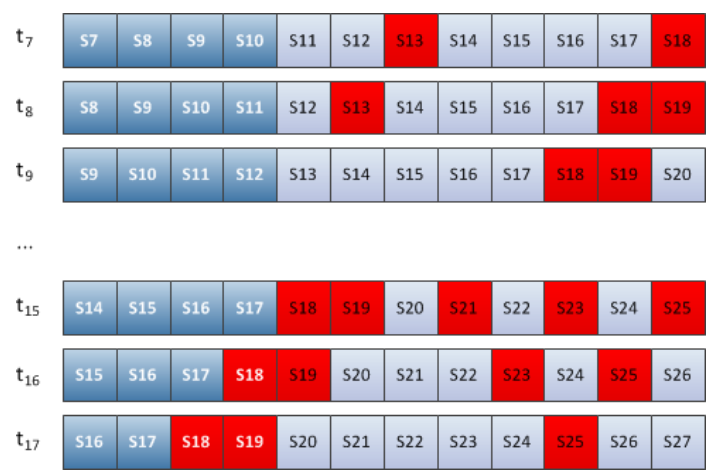

b)

Fig. 16. Example of buffering in the proxy

Finally, it is worth mentioning that more segment recovery techniques and priority policies can be applied. In fact, the use of an on-board proxy instead of a simple buffer opens up a new world of possibilities, including reencoding video segments, addition of FEC (Forward Error Correction) techniques to improve wireless transmission, and other mechanisms that fall out the scope of this paper.

\section{Conclusion}

This paper has presented a system for the reception of near real-time TV/radio services in mobility using 4G. The system is based on the existence of a server on the cloud in charge of transcoding the video flows received through DVB in order to be sent using DASH through a $4 \mathrm{G}$ link. The main component in the architecture is the proxy shipped in the vehicle, which includes a buffering module to manage the video streams and perform recovering tasks.

Moreover, the paper performs multiple evaluations of the proposed system in two main scenarios, using simulated channels and performing measurements in an on-board implementation. Simulation results reflect that the proxy performs better than the live player in channels with variable bandwidth. Regarding on-board tests, the different evaluations carried out have proved that the use of the proxy in the shipped server reduces considerably both the number and the duration of the interruptions. Therefore, the buffer size is important to absorb the interruptions produced by the lack of connectivity, losses or packet delays, although the downside of using the proxy is the difference between real-time and the timeline in on-board players.

For the scenarios under study, the proxy reduces the duration of the interruptions a $95.7 \%$ compared to a direct reproduction. Furthermore, it is important to take into account that the vehicle has crossed urban, rural and interurban areas with orographic differences and, therefore, with different coverage and channel bandwidth. Hence, results show that the improvement using the proxy can vary considerably depending on the scenario. The architecture might be improved by developing an algorithm to anticipate the channel conditions based on location and previous channel measurements. This way the proxy may adapt the buffer length to foresee the expected losses and coverage leaks.

Finally, it is worth highlighting that one of the main contributions of the paper is a real implementation of the whole system and the configuration of the testbed, which is used to carry out the assessment of the proposal. As a result, we have implemented a real application of a proxy service, which improves the performance of live video streaming in several ways. First, we have shown the reduction in the impact of signal loss in video playback during 
mobility scenarios, reducing interruptions and improving the quality of user experience. Additionally, from a practical point of view, the fact of using this approach could reduce the overall bandwidth consumed in the vehicle (and the related cost), since only one flow is requested from the cloud and redistributed to all clients on-board instead of being every client who requests their own flow. Another contribution to be considered is the fact that we have used a real vehicle with an actual $4 \mathrm{G}$ connection. Therefore, besides the simulated environments, we managed to carry out a proof of concept in real scenarios, with the practical implications that this entails.

As a future work, as we explained in previous section, we plan to implement a quality selection algorithm in the proxy, so that it will have the possibility to minimize playback interruptions when a signal loss occurs by reducing the quality of the requested segments. This will optimize the bandwidth usage and reduce video interruptions in most cases, but at the expense of reducing the playback quality.

\section{Acknowledgements}

This work is supported by the Centro para el Desarrollo Tecnológico Industrial (CDTI) from the Government of Spain under the project "Plataforma avanzada de conectividad en movilidad" (CDTI IDI-20150126) and the project "Desarrollo de nueva plataforma de entretenimiento multimedia para entornos náuticos" (CDTI TIC-20170102).

\section{References}

1. Cisco (2017) Cisco VNI Forecast and Methodology, 2016-2021. White paper.

2. Acharya S, Alonso R, Franklin M, Zdonik S (1995), Broadcast disks: data management for asymmetric communication environments. Proc. of the ACM SIGMOD Conf. on Manag. of Data, San Jose, California, USA: 199-210. doi: 10.1145/223784.223816.

3. Zhiqi G, Songyu Y, Wenjun Z (2004) Using object multiplex technique in data broadcast on digital CATV channel. IEEE Trans. on Broadcast. 50-2:113-119. doi: 10.1109/TBC.2004.828365

4. Erman J, Gerber A, Hajiaghayi M, Pei D, Sen S, Spatscheck O (2011) To cache or not to cache - The 3G Case. IEEE Internet Comput. 15-2:27-34. doi: 10.1109/MIC.2010.154.

5. Woo S, Jeong E, Park S, Lee J, Ihm S, Park K (2013) Comparison of caching strategies in modern cellular backhaul networks. Proc. of the ACM MobiSys, Taipei, Taiwan:319-332. doi: $10.1145 / 2462456.2464442$.

6. Ramanan B A, Drabeck L M, Haner M, Nithi N, Klein T E, Sawkar C (2013) Cacheability analysis of HTTP traffic in an operational LTE network. Wirel. Telecommun. Symp., Phoenix, AZ, United States. doi: 10.1109/WTS.2013.6566245.

7. Malandrino F, Chiasserini C F, Kirkpatrick S (2017) The impact of vehicular traffic demand on 5G caching architectures: A data-driven study. Veh. Comm. 13-20. doi: 10.1016/j.vehcom.2016.11.007.

8. Samdanis K, Taleb T, Schmid S (2012) Traffic offload enhancements for eUTRAN. IEEE Commun. Surv. \& Tutor. 14-3:884 896. doi: 10.1109/SURV.2011.072711.00168.

9. Taleb T, Hadjadj-Aoul Y, Schmid S (2011) Geographical location and load based gateway selection for optimal traffic offload in mobile networks. Proc. of the IFIP Networking, Valencia, Spain:331-342. doi: 10.1007/978.3.642.20757.0.26.

10. Chuang M C, Chen M C (2015) A mobile proxy architecture for video services over high-speed rail environments in LTE-A networks. IEEE Syst. J. 9-4:1264-1272. doi: 10.1109/JSYST.2014.2354435. 
11. 3GPP (2012) Requirements for Further Advancements for E-UTRA (LTE-Advanced), Sophia-Antipolis Cedex, France, TR 36.913 V8.0.0, Release 11.

12. Hu H, Zhu X, Wang Y, Pan R, Zhu J, Bonomi F (2013) Proxy-based multi-stream scalable video adaptation over wireless networks using subjective quality and rate models. IEEE Trans. on Multim. 157:1638-1652. doi: 10.1109/TMM.2013.2266092.

13. Chakareski J (2014) Wireless streaming of interactive multi-view video via network compression and path diversity. IEEE Trans. on Comm. 62-4:1350-1357. doi: 10.1109/TCOMM.2014.022314.120890.

14. Zhao M, Gong X, Liang J, Wang W, Que X, Cheng S (2015) QoE-driven cross-layer optimization for wireless dynamic adaptive streaming of scalable videos over HTTP. IEEE Trans. on Circuits and Syst. for Video Technol. 25-3: 451-465. doi: 10.1109/TCSVT.2014.2357094

15. Digital Video Broadcasting (DVB) (2004) Transmission System for Handheld Terminals (DVB-H), ETSI EN 302304 v1.1.1.

16. Digital Video Broadcasting (DVB) (2009) System specifications for satellite services to handheld devices (SH) below $3 \mathrm{GHz}$, ETS ITS 102585 v1.2.1.

17. 3GPP (2013) Universal Mobile Telecommunications System (UMTS); LTE; Multimedia Broadcast/Multicast Service (MBMS); Protocols and codecs (release 11), ETSI TS 126346 v11.5.0.

18. 3GPP (2017) Evolved Universal Terrestrial Radio Access (E-UTRA) and Evolved Universal Terrestrial Radio Access Network (E-UTRAN); Overall description; Stage 2 (release 8).

19. Bangerter B, Talwar S, Arefi R, Stewart K (2014) Networks and devices for the 5G era. IEEE Comm. Mag., 52-2:90-96, doi: 10.1109/MCOM.2014.6736748.

20. GSMA Intelligence (2018) Mapping worldwide 4G-LTE network launches. http://gsmaintelligence.com/analysis/2013/08/dashboard-mapping-worldwide-4g-lte-network-launchesaugust-2013/399. Accessed 21 March 2018.

21. Informa Telecoms \& Media (2018) LTE Spectrum Strategies and Forecasts to 2018, 3rd ed. http://www.informa.com/Media-centre/Press-releases—news/Latest-News/Informa-Telecoms-andMedia-Huawei-and-Ericsson-dominate-LTE-contracts-as-deployments-accelerate. Accessed 21 March 2018.

22. Evans B G (2014) The role of satellites in 5G. Proc. of the ASMS/SPSC, Livorno:197-202. doi: 10.1109/ASMS-SPSC.2014.6934544.

23. Lau C P, Alabbasi A, Shihada B (2016) An efficient live TV scheduling system for 4G LTE broadcast. IEEE Syst. J. 11-4:2737-2748. doi: 10.1109/JSYST.2015.2493180.

24. Kumar S, Sarkar A, Sur A (2017) A resource allocation framework for adaptive video streaming over LTE. J. of Netw. and Comp. App. 97:126-139. doi: 10.1016/j.jnca.2017.08.015.

25. Yrjöla S, Huuhka E, Talmola P, Knuutila T (2017) Coexistence of Digital Terrestrial Television and 4G LTE mobile network utilizing supplemental downlink concept: a real case study. IEEE Trans. on Veh. Technol. 66-6:5422-5434. doi: 10.1109/TVT.2016.2628088.

26. Tabrizi F M, Peters J, Hefeeda M (2013) Dynamic control of receiver buffers in mobile video streaming systems. IEEE Trans. on Mob. Comput. 12-5:995-1008. Doi: 10.1109/TMC.2012.56.

27. ISO/IEC 23009-1 (2012) Dynamic adaptive streaming over HTTP (DASH) - Part 1: media presentation description and segment formats. 
28. International Telecommunication Union (ITU-T) (2017) Parametric bitstream-based quality assessment of progressive download and adaptive audiovisual streaming services over reliable transportRecommendation ITU-TP.1203.

29. Robitza W, Göring S, Raake A, Lindegren D, Heikkilä G, Gustafsson J, List P, Feiten B, Wüstenghage U, Garcia M N, Yamagishi K, Broom S (2018) HTTP adaptive streaming QoE estimation with ITU-T rec. P. 1203: open databases and software. Proc. of the ACM Multim. Syst. Conf. (MMSys) Amsterdam, Netherlands, 466-471.

30. International Telecommunication Union (2016) Mean opinion score (MOS) terminology. ITU-T P.800.1, Series P: terminals and subjective and objective assessment methods, Methods for objective and subjective assessment of speech and video quality.

31. Ghadiyaram D, Pan J, Bovik A C (2019) A subjective and objective study of stalling events in mobile streaming videos. IEEE Trans. on Circuits and Syst. for Video Technol. 29-1:183-197. doi: 10.1109/TCSVT.2017.2768542. 